\title{
Pregnancy outcome in women with antiphospholipid syndrome on low-dose aspirin and heparin: a retrospective study
}

T. Naru, ${ }^{7}$ R.S. Khan ${ }^{7}$ and R. Ali ${ }^{1}$

$$
\text { طتائج الحمل لدى المصابات بمتلازمة مضادات الفوسفولبيد اللاتي يعالجن بجرعة منخفضة من الأسبرين والهيبارين: دراسة }
$$

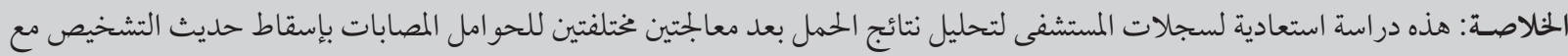

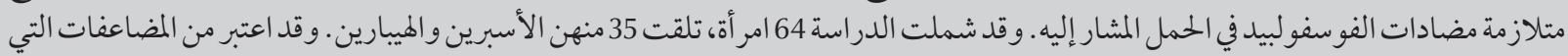

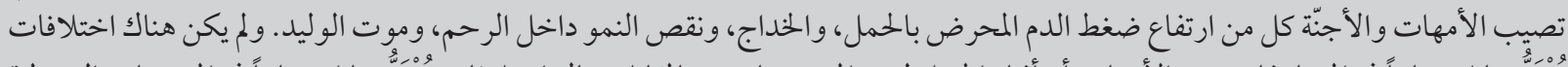

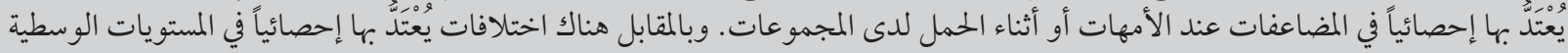

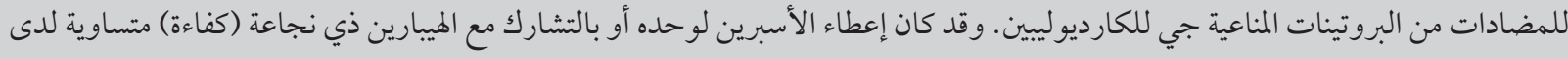

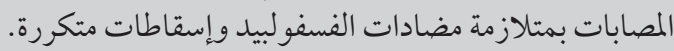

ABSTRACT This retrospective review of hospital records analysed pregnancy outcome with 2 different treatments for women with recurrent miscarriage diagnosed with antiphospholipid syndrome in the index pregnancy. Of 64 women, 29 had received aspirin and 35 aspirin plus heparin. Pregnancy-induced hypertension, prematurity, intrauterine growth restriction and neonatal death were considered as maternal and fetal complications. There were no significant differences in antenatal and maternal complications between the groups. However, there were significant differences in mean anticardiolipin IgG antibody levels. Aspirin alone or in combination with heparin was equally efficacious in women with antiphospholipid syndrome and recurrent miscarriage.

Issue de la grossesse de femmes présentant un syndrome des anticorps antiphospholipides traité par I'aspirine à faible dose et par l'héparine : une étude rétrospective

RÉSUMÉ Cette étude rétrospective des dossiers hospitaliers a analysé l'issue de la grossesse chez des femmes ayant fait des fausses couches à répétition, chez lesquelles un syndrome des anticorps antiphospholipides a été diagnostiqué et qui ont reçu deux traitements différents. Sur 64 femmes, 29 d'entre elles avaient reçu de l'aspirine, tandis que 35 étaient traitées avec de l'aspirine et de l'héparine. L'hypertension induite par la grossesse, la prématurité, les retards de croissance intra-utérine et les décès néonatals ont été considérés comme des complications maternelles et fotales. Aucune différence significative en termes de complications prénatales et maternelles n'a été constatée entre les deux groupes. Cependant, des différences importantes ont été observées dans les taux moyens d'anticorps anticardiolipine lgG. L'aspirine seule possède la même efficacité que l'aspirine associée à l'héparine chez les femmes présentant un syndrome des anticorps antiphospholipides et ayant fait des fausses couches à répétition. 


\section{Introduction}

Antiphospholipid antibody syndrome (APS) is a disorder characterized by the presence of medium to high levels of lupus anticoagulant antibodies (LAC) and anticardiolipin antibodies (aCL) - the so-called antiphospholipid antibodies (aPL). APS was first described in the early 1980s in patients with systemic lupus erythematosus $[1,2]$ and is termed primary APS when it occurs in otherwise healthy people [3]. APS is associated with arterial and venous thrombosis and both early pregnancy loss and fetal death in advanced pregnancy $[1-4]$. While placental thrombosis and infarction are common findings in aPL-related intrauterine fetal deaths [4], thrombosis is not a universal finding and alternative mechanisms of pregnancy loss have been sought $[5,6]$.

Prospective clinical studies have confirmed that aPL are risk factors for pregnancy loss, both in patients with systemic lupus erythematosus [5] and in healthy nulliparous women [7]. In women with a history of 3 or more consecutive pregnancy losses persistently positive tests for aPL were found in up to $15 \%$, and in these cases, subsequent fetal loss rates of up to $60 \%-90 \%$ were noted without specific treatment $[8,9]$. aPL are also associated with a high incidence of pre-eclampsia, intrauterine growth restriction (IUGR), fetal distress and premature delivery $[10,11]$.

A number of studies have evaluated the efficacy of treatment with low-dose aspirin, prednisolone, unfractionated low-molecular weight heparin and most recently intravenous gammaglobulin, either alone or in various combinations. However, the findings have not been consistent $[12,13]$. Low-dose aspirin in combination with heparin was demonstrated in 2 randomized controlled trials to lead to a significant improvement in the live birth rate $[14,15]$.

This study aimed to determine the pregnancy outcome in women with
APS and recurrent pregnancy loss who were treated with aspirin alone or aspirin in combination with heparin during the index pregnancy.

\section{Methods}

\section{Sample and setting}

This study was conducted at the Aga Khan University Hospital in Karachi, Pakistan, a tertiary care unit with an average of 3500 deliveries per year. A dedicated outpatient service caters to high-risk obstetric care, early pregnancy complications and investigations including Doppler ultrasound facilities in the fetomaternal unit. The nursing staff provides regular antenatal sessions as well as patient education and training for self-administered injections.

The criteria for entry to the study were women who suffered 2 or more 1st or 2nd trimester fetal deaths or at least 1 intrauterine death or neonatal death and 1 of the following laboratory findings before or during the index pregnancy: aCL > 5 GPL [IgG phospholipid units] or > 5 MPL [IgM phospholipid units].

\section{Data collection}

The study was a retrospective analysis of women who voluntarily chose different treatments. From January 1994 to December 2003 the data were retrieved from a data indexing and coding system based on the International classification of diseases, 9th edition. Of 84 women identified, 20 were excluded due to incomplete documentation and the remaining 64 women were divided into 2 groups: group A $(n=29)$ had received low-dose aspirin $(75 \mathrm{mg}$ once daily orally) while group B $(n=35)$ had received aspirin ( $75 \mathrm{mg}$ once daily orally) plus heparin (5000 IU subcutaneously twice daily) (group B) during the index pregnancy. The group of patient on aspirin treatment alone were those who had refused heparin, as injections were not acceptable to them. Aspirin had been commenced from the start of the pregnancy either after a positive pregnancy test or presence of cardiac activity on ultrasound scan until the 34th week of gestation, while in group $\mathrm{B}$ heparin had been started in the $2 \mathrm{nd}$ trimester and continued until the 36th week of gestation.

Most of the women were assessed before pregnancy when they came to the clinic for counselling regarding more than 2 pregnancy losses (intrauterine and/or neonatal deaths). Women were monitored fortnightly during the 1st trimester for hypertension and thrombocytopenia. Fetal monitoring was started from 24-26 weeks with monthly growth scans until delivery. Two-weekly scans were performed if severe early onset of IUGR was suspected along with umbilical artery Doppler studies. The timing of delivery was assessed individually on the basis of current and past obstetric history and the onset of complications, e.g. pre-eclampsia, IUGR or abnormal Doppler results. Women whose pregnancy reached 37 completed weeks gestation were either induced or delivered by elective caesarean section. In women who received heparin during pregnancy, the treatment was used until the end of the hospital stay and lowdose aspirin was continued until 6-8 weeks postpartum.

\section{Analysis}

The data were analysed using SPSS, version 10. Independent sample $t$-tests were used to compare 1st, 2nd and 3rd trimester pregnancy losses, neonatal deaths and status of aPL. Chi-squared and Fisher exact tests were used for the association between maternal age, gestational age, birth weight, status of aPL and pregnancy outcome. A $P$-value $<0.05$ was considered significant.

\section{Results}

A total of 64 women with APS and recurrent miscarriage were included in 


\begin{tabular}{lccc}
\hline \multicolumn{1}{l}{ Table 1 Age and pregnanacy characteristics of women treated with aspirin or aspirin plus heparin $(\boldsymbol{n}=\mathbf{6 4})$} & Group B \\
\hline Characteristic & Group A & $\boldsymbol{P}$-value \\
& Aspirin $(\boldsymbol{n}=29)$ & Aspirin + heparin $(\boldsymbol{n}=35)$ \\
Maternal age (years) & Mean (SD) & Mean (SD) & 0.575 \\
1st and 2nd trimester pregnancy loss (No.) & $28.8(5.3)$ & $29.6(5.2)$ & 0.726 \\
3rd trimester pregnancy loss (No.) & $2.28(1.13)$ & $2.43(2.10)$ & 0.300 \\
Neonatal death (No.) & $0.21(0.41)$ & $0.34(0.39)$ & 0.547 \\
\hline
\end{tabular}

$S D=$ standard deviation .

the study: 29 women in group A used only low-dose aspirin and 35 women in group $B$ received low-dose aspirin plus heparin. Tables 1 and 2 show the demographic details and antiphospholipid antibody status of the women. There were no significant differences between the groups with respect to maternal age, number of previous 1st, 2nd and 3rd trimester pregnancy losses and neonatal deaths.

There was a significant difference in the mean concentrations of aCL IgG titres between group A and group B [8.88 (SD 7.17) GPL versus 15.88 (SD 13.77) GPL] $(P=0.016)($ Table 2$)$. The mean concentrations of aCL IgM titres were not significantly different between the groups [10.73 (SD 7.19) MPL versus 12.30 (SD 7.61) MPL] ( $P$ $=0.403$ ).

There were no significant differences between groups $\mathrm{A}$ and $\mathrm{B}$ in the rates of pre-eclampsia $[6 / 29$ (20.7\%) versus 10/35 (28.6\%)], preterm births [9/29 (31.0\%) versus $12 / 35(34.3 \%)]$ and IUGR $[5 / 29(17.2 \%)$ versus $8 / 35$ (22.9\%)] (Table 3).

The mean gestational age and the neonatal birth weight were not significantly different between the groups; the mean gestational ages at delivery in groups $\mathrm{A}$ and $\mathrm{B}$ were 35.9 (SD 4.1) months and 35.6 (SD 3.6) months respectively. The mean birth weights in groups A and B were 2.47 (SD 0.85) $\mathrm{kg}$ and 2.36 (SD 0.77) kg respectively. There were 6 neonatal deaths (20.7\%) in group A and 3 (8.3\%) in group B. One baby in group A died due to congenital heart disease (Table 3). None of the women developed a thromboembolic or cerebrovascular event.

\section{Discussion}

APS is widely recognized as a risk factor for numerous obstetric complications, including recurrent miscarriage, IUGR, pre-eclampsia, fetal death and preterm labour. Since its original description, APS has emerged as the most important treatable cause of recurrent miscarriage [2]. The prevalence of aPL in recurrent miscarriage is approximately $15.5 \%[9,16]$. The risk of subsequent pregnancy loss in women with aPL and previous pregnancy loss is unknown but probably exceeds $60 \%$ [10].

A variety of treatment regimens have been used, both single agents and combinations, to improve the poor live birth rates among these women, with live births reported from 30\% to 100\% of pregnancies [1]. However, treatment of pregnant aPL-positive women to improve pregnancy outcome remains completely empirical. The paucity of data from large well-designed trials of different management options in comparable groups of pregnant women with this complex disease contributes to the difficulty in formulating recommendations for managing these pregnancies. The approach is guided by observational studies, personal knowledge and the opinions of others experienced in the management of such women.

Our study investigated women with APS in the index pregnancy treated with either aspirin alone or aspirin and heparin together. Analysis of the data did not reveal any statistically significant differencebetween the treatmentgroups in terms of live birth rate or the antenatal complication rates. Preterm deliveries were experienced by $31.0 \%$ of women in group A compared with $34.3 \%$ in group B, while $17.2 \%$ and $22.9 \%$ of women suffered IUGR in groups A and B respectively. Similarly, severe pre-eclampsia was observed in $20.7 \%$ and $28.6 \%$ of

\begin{tabular}{lccc}
\hline Table 2 Antiphospholipid antibody levels of women treated with aspirin or aspirin plus heparin $(\boldsymbol{n}=\mathbf{6 4})$ & Group B & $\boldsymbol{P}$-value \\
\hline Antibody levels & Group A & Aspirin $(\boldsymbol{n}=29)$ & Aspirin + heparin $(\boldsymbol{n}=35)$ \\
& Mean (SD) & Mean (SD) & \\
& $8.88(7.17)$ & $15.88(13.77)$ & 0.016 \\
Anticardiolipin IgG (GPL) & $10.73(7.19)$ & $12.30(7.61)$ & 0.403 \\
Cardiolipin IgM (MPL)
\end{tabular}

$S D=$ standard deviation $; G P L=\lg G$ phospholipid units; $M P L=\lg M$ phospholipid units. 


\begin{tabular}{|c|c|c|c|}
\hline Maternal and fetal outcome & Group A & Group B & $P$-value \\
\hline & Aspirin $(n=29)$ & Aspirin + heparin $(n=35)$ & \\
\hline Pre-eclampsia (No.) & 6 & 10 & 0.568 \\
\hline Preterm deliverya (No.) & 9 & 12 & 0.498 \\
\hline Small-for-gestational age baby (No.) & 5 & 8 & 0.757 \\
\hline Gestational age [Mean (SD) weeks] & $35.9(4.2)$ & $35.6(3.6)$ & 0.720 \\
\hline Birth weight [Mean (SD) g] & $2.5(0.9)$ & $2.4(0.8)$ & 0.581 \\
\hline \multicolumn{4}{|l|}{ Neonatal death (No.) } \\
\hline Yes & $6^{\mathrm{b}}$ & 3 & 0.152 \\
\hline No & 23 & 32 & \\
\hline
\end{tabular}

${ }^{a}$ At 26-37 weeks.

${ }^{b} 7$ baby had congenital heart disease.

$S D=$ standard deviation .

the women respectively. These results are comparable to international data $[11,12,16,17]$.

Stone et al. analysed the outcome of pregnancies after introduction of a standard protocol in 33 patients with APS and achieved a live birth rate of 91\% in women with primary APS even in those with significant past pregnancy morbidity with or without thrombosis. The outcome in the index pregnancy was influenced the most by past pregnancy outcome [18]. Branch and Khamashta pointed out that treatment of APS must be individualized and related to previous pregnancy complications [19].

A prospective study of aPL-positive pregnant women with at least 3 spontaneous consecutive miscarriages recorded $44 \%$ of live births in women assigned to low-dose aspirin alone and $80 \%$ for those treated with aspirin plus subcutaneous heparin twice daily [14]. A comparable trial also found aspirin alone inferior to aspirin plus heparin ( $42 \%$ versus $71 \%$ live births) [15]. In view of the heterogeneous definition of aPL in the literature,
Kutteh excluded women with LAC [14], whereas in the study of Rai et al., $80 \%$ of the patients had LAC in the absence of aCL [15]. Meanwhile, Farquharson et al. found the birth rate to be similar in both groups ( $72 \%$ with aspirin alone compared with $78 \%$ when heparin was added to the regimen) [20]. The uncertainty as to whether heparin is always needed comes from this uncontrolled prospective study with $91 \%$ live births with low-dose aspirin alone in aPL-positive pregnant women, who had a minimum of 2 spontaneous abortions and a history of only $6 \%$ live births [18]. Differences in the inclusion and exclusion criteria used in different studies are a major cause of such discordant findings [21].

Our study suggests that the women with aPL and recurrent pregnancy loss can be treated effectively with low-dose aspirin alone. As this study did not include untreated controls, we cannot exclude the possibility that aspects of obstetric care other than the treatment per se influenced pregnancy outcome.

An important finding in this study was the statistical significant difference in the $\operatorname{IgG}$ values between the aspirin group and the aspirin plus heparin group $(P=0.016)$. The maternal and fetal complications in the 2 groups were similar. We cannot exclude the possibility of a better outcome in the aspirin and heparin group due to the addition of heparin. The persistent presence of IgG and IgM aCL needs to be clinically monitored during pregnancy for optimal outcome. This may have some implications for further study and counselling of these women.

As complications still occur and the exact pathogenetic mechanism of these events in APS remains unclear, further randomized trials are required to determine the optimum regime for the management of APS pregnancies. Among combination therapies, aspirin together with heparin is currently one of the best treatments offered, with the fewest adverse drug effects. The possibility of adverse effects with heparin therapy for a longer duration, and the cost implications for low resource areas needs consideration.

\section{References}

1. Petri M. Pathogenesis and treatment of the antiphospholipid antibody syndrome. Medical clinics of North America, 1997, 81:151-77.

2. Wilson WA et al. International consensus statement on preliminary classification criteria for definite antiphospholipid syndrome: report of an international workshop. Arthritis and rheumatism, 1999, 42:1309-11.

3. Asherson RA et al. The "primary" antiphospholipid syndrome: major clinical and serological features. Medicine (Baltimore), 1989, 68:366-74. 
4. Out $\mathrm{HJ}$ et al. Histopathological findings in placentae from patients with intra-uterine fetal death and antiphospholipid antibodies. European journal of obstetrics and gynecology, 1991, 41:179-86.

5. Di Simone $\mathrm{N}$ et al. Antiphospholipid antibodies regulate the expression of trophoblast cell adhesion molecules. Fertility and sterility, 2002, 77:805-11.

6. Di Simone $\mathrm{N}$ et al. In vitro effect of antiphospholipid antibodycontaining sera on basal and gonadotrophin releasing hormone dependent human chronic gonadotrophin released by cultured trophoblast cells. Placenta, 1995, 16:75-83.

7. Love PE, Santoro SA. Antiphospholipid antibodies: anticardiolipin and the lupus anticoagulant in systemic lupus erythematosus (SLE) and in non-SLE disorders. Prevalence and clinical significance. Annals of internal medicine, 1990, 112:682-98.

8. Lynch A et al. Antiphospholipid antibodies in predicting adverse pregnancy outcome. A prospective study. Annals of internal medicine, 1994, 120:470-5.

9. Rai RS et al. High prospective fetal loss rate in untreated pregnancies of women with recurrent miscarriage and antiphospholipid antibodies. Human reproduction, 1995, 10:3301-4.

10. Branch DW et al. Outcome of treated pregnancies in women with antiphospholipid syndrome: an update of the Utah experience. Obstetrics and gynecology, 1992, 80:614-20.

11. Lima F et al. A study of sixty pregnancies in patients with the antiphospholipid syndrome. Clinical and experimental rheumatology, 1996, 14:131-6.

12. Stone S, Poston L. Antiphospholipid antibody syndrome in pregnancy: onset to outcome. Fetal and maternal medicine review, 2004, 15:273-97.
13. Empson M et al. Recurrent pregnancy loss with antiphospholipid antibody: a systemic review of therapeutic trials. Obstetrics and gynecology, 2002, 99:135-44.

14. Kutteh WH. Antiphospholipid antibody associated recurrent pregnancy loss: treatment with heparin and low dose aspirin is superior to low dose aspirin alone. American journal of obstetrics and gynaecology, 1996, 174:1584-9.

15. Rai R et al. Randomised controlled trial of aspirin plus heparin in pregnant women with recurrent miscarriage associated with antiphospholipid antibodies. British medical journal, 1997, 314:253-7.

16. Backos $\mathrm{M}$ et al. Pregnancy complications in women with recurrent miscarriage associated with aPL treated with low dose aspirin and heparin. British journal of obstetrics and gynaecology, 1999, 106:102-7.

17. Vinatier D et al. Antiphospholipid syndrome and recurrent miscarriages. European journal of obstetrics, gynecology, and reproductive biology, 2001, 96:37-50.

18. Stone $\mathrm{S}$ et al. Primary antiphospholipid syndrome in pregnancy: an analysis of outcome in a cohort of 33 women treated with a rigorous protocol. Journal of thrombosis and haemostasis, 2005, 3(2):240-2.

19. Branch DW, Khamashta MA. Antiphospholipid syndrome: obstetric diagnosis, management and controversies. Obstetrics and gynecology, 2003, 101(6):1333-44.

20. Farquharson RG, Quenby S, Greaves M. Antiphospholipid syndrome in pregnancy: a randomized, controlled trial of treatment. Obstetrics and gynecology, 2002, 100:408-13.

21. Balasch J et al. Low-dose aspirin for prevention of pregnancy losses in women with primary antiphospholipid syndrome. Human reproduction, 1993, 8:2234-9.

\section{Mental health aspects of women's reproductive health. A global review of the literature}

This book has reviewed the research undertaken on a broad range of reproductive health issues and their mental health determinants/consequences over the last 15 years from both high- and low-income countries. Evidence from peerreviewed journals has been used wherever possible but has been augmented with results of a specific survey initiated to gather state of the art information on reproductive and mental health issues from a variety of researchers and interested parties. Valuable data from consultant reports, national programme evaluations and postgraduate research work was also compiled, analyzed and synthesized.

Further information about this and other WHO publications can be found at: http://www.who.int/publications/en/ 